\title{
XI.
}

\section{Ueber ein Myosarcoma striocellulare des Nierenbeckens und des Ureters.}

\author{
Von Prof. Dr. Ribbert, \\ I. Assistenten am pathologischen lustitut zu Bonn.
}

Sarcome der Nieren mit quergestreiften Muskelfasern sind von Eberth ${ }^{1}$ ), Cohnheim ${ }^{2}$ ), Marchand ${ }^{3}$ ), Landsberger und Cohnheim ${ }^{4}$ ), Kocher und Langhans ${ }^{5}$ ), Huber und Boström ${ }^{6}$ ) und von Brosin ${ }^{7}$ ) beobachtet worden. Das grosse Interesse, welches diese Tumoren in mehrfacher Hinsicht darbieten, würde die Beschreibung eines neuen Falles auch dann rechtfertigen, wenn er nicht, wie der von mir untersuchte, nach verschiedenen Richtungen ein eigenartiges Verhalten zeigte.

Es handelte sich um ein 4jähriges Mädchen, bei welchem in den letzten Monaten eine zunehmende Auftreibung des Bauches bemerkt wurde. Krankhafte Erscheinungen aus den ersten Iuebensjahren des Kindes wusste die Mutter nicht anzugeben. Die Diagnose lautete auf Tumor des Abdomens, wahrscheinlich von der rechten Niere ausgehend. Die Operation wurde von Herm Privatdocenten Dr. Witzel gemacht, der genauere Mittheilungen darïber in der "Deutschen Zeitschr. f. Chirurgie" veröffentlichte. Ich beschreibe zunächst die dem pathologischen Institut übermittelte Geschwulst nach ihrem makroskopischen Verhalten.

Der Tumor repräsentirt eine durch knollige Geschwulstmassen ausgefüllte Cyste. Der Inhalt drängt sich aus einem grossen unregelmässigen Einriss

1) Dieses Archiv Bd. 55. S. 518.

2) Ibid. Bd. 65. S. 64 .

3) Ibid. Bd. 73. S. 289.

4) Berliner klin. Wochenschr. 1877. No. 34 .

5) Deutsche Zeitschr. f. Chir. Bd.9. S. 312.

$\left.{ }^{6}\right)$ Deutsches Archiv f. klin. Med. Bd, 23. S. 205.

7) Dieses Archiv Bd. 96. S. 453. 
der Wandung nach aussen vor. Die Oberfläche wird gebildet durch ein uneben fetziges Bindegewebe, welches sich auf der Vorderfläche als kapselartiger Ueberzug abtrennen lässt. Unter diesem kommt dann in einer Ausdehunng von $23: 11 \mathrm{~cm}$ die vollkommen glatte Oberfäche der Niere zut Vorschein, mit unregelmässig buchtigem Rande, der sich aber scharf gegen die übrige Wand der Cyste absetzt. Das Organ nimmt etwa den 3. bis 4. Theil der Gesammtoberfläche ein. Es ist von innen her plattgedrückt, über den Inhalt der Cyste herübergespannt und dadurch in dem angegebenen Umfange über eine grössere Fläche ausgedebnt. Es stellt so nur noch einen platten Körper dar, der, wie Durchschnitte feststellen, böchstens $5 \mathrm{~mm}$, vielfach aber nur $1 \mathrm{~mm}$ dick ist. Rinde und Mark sind nur an den dicken Stellen noch undeutlich von einander abzugrenzen. Die übrige Wand der Cyste besteht aus einem weichen, stellenweise faserigen Gewebe in wechselnder bis zu $3 \mathrm{~cm}$ gehender Dicke. Letztere Partien springen in den Cystenraum flach buckelig vor. In den Bereich dieses nicht von Niere bedeckten Abschnittes der Wand fällt der erwähnte Einriss, am Rande desselben in einer Winkelstelle hängt ein $2 \mathrm{~cm}$ langer Kanal, dessen Innenfläche in die des Cystenmumes continuiplici übergebt und der offenbar den bei der Operation durehschnittenen Anfangstheil des Ureters darstellt. Die Schleimhaut desselben lässt sich nur eine Strecke weit in die Cyste verfolgen, dann hört sie zackig, offenbar eingerissen auf und weiterhin finden sich nur noch einzelne gleichfalls zackige isolirte Abschnitte desselben.

Die Cyste ist also zweifellos das mächtig erweiterte Niereabecken. Es wird angefült durch viele Tumoren, die aus sebliesslich da der Wand aufsitzen, wo sie von Nierengewebe bedeckt ist. Es sind gestielte Geschwülste ron wechselndem Umfange. Einer der Polypen ist über faustgross, derb, flachknollig, mit nicht ganz fingerdickem Stiel, ein zweiter, im Ganzen etwas kleiner, ist sehr weich, eingerissen, zerfetzt und sitzt mit breiter mehrfach getheilter Basis auf, ausserdem ist noch eine ganze Reihe kleinerer Polypen mit dünnerem Stiel vorhanden.

Sehen wir uns nun weiterhin die Beziehungen des Geschwulstgewebes zur Niere genaner an, so ergiebt sich nach Anlegung eines Schnittes, der einen nussgrossen Polypen senkrecht zur Cystenwand bis zu deren Oberfläche durchtrennt, dass der kurze und dünne Stiel desselben nochmals in einen fast gleichgrossen Geschwulstknoten übergeht, der in einer ringsherumgehenden von Nierengewebe gebildeten Höhle liegt. Es handelt sich hier offenbar um einen Kelch des Nierenbeckens, der durch den Tumor zu einer rundlichen Höhle ausgedehnt wurde. Gleich neben diesem, durch 2 mm dickes Nierengewebe davon getrennt, befindet sich ein zweiter in gleicher Weise erweiterter Calix, dessen Tumor ebenfalls halsförmig eingeschnürt ist und in den Cystenraum mehrfach gelappt vorspringt. Auch der oben erwähnte grösste Knoten ist in gleicher Weise befestigt, während unter den einzelnen Abschnitten der anderen grösseren Geschwulstmasse nur einer deutlich aus einem Kelch hervorkommt. Die Markkegel sind in den betreffenden Calices durch 
die Tumoren vollkommen abgeflacht und nur schwer von der Nierenrinde abzugrenzen.

Die so bis auf die halsförmige Einschnürung ringsum eingeschlossenen Theile der Polypen lassen sich obne Iuhe ausschälen, dabei zerreisst nur ein dünnes lockeres Gewebe mit einzelnen etwas festeren Strängen (Gefässen). Diese weiche Bindesubstanz bildet die nur sebr dünne trennende Lage zwischen Tumor und Niere.

Die Unterfläche der Niere ist nicht in ganzer Ausdebnung mit polypösen Geschwülsten besetzt, es bleibt vielmehr peripheriseh ein bis zu $2 \mathrm{~cm}$ breiter Abschnitt frei. Hier ist sie entweder nur von einer geringen Menge streifigen Gewebes, oder von einer dicken $1-2 \mathrm{~cm}$ hohen Geschwulstmasse bedeckt, welche sich in die übrige Cystenwand continuirlich fortsetzl und dort, wie bereits erwähnt, innen noch kleinere und grössere Fetzen von Nierenbeckenschleimhant trägt. Das Tumorgewebe setzt sich an Rande der Niere auch noch eine Strecke weit, aber rasch an Dicke abnehmend, in die Nierenkapsel fort, die auf der übrigen Oberfläche des Organs kaum dicker als normal und von Geschwulst volltommen frei ist. So ist der Saum der Niere vielfach beiderseits in Tumormasse eingelagert, aber auf der Schnittfäche kann man ihren zugespitzten Rand noch deutlich abgrenzen, zumal auf der Aussenfläche, da sich hier die durch Geschwulstgewebe verdickte Kapsel ebenso leicht ablösen lässt wie auf der übrigen Niere.

Diese makroskopischen Verbältnisse stellen zweifellos fest, dass die Geschwulst nicht aus der Niere, sondern aus der Wandung des Nierenbeckens sich entwickelt hat. Im Bereich der Kelche ist von letzterer nur noch die dünne lockere Gewebslage erhalten, die auch in der Norm die eigentliche Wand von der Niere trennt, im Uebrigen ist sie für das freie Auge ganz in den Tumor aufgegangen. Ausserbalb des von der Niere bedeckten Abschnittes der Cyste sind fast ausschliesslich die lockeren äusseren Schichten der Beckenwand ergriffen, aber die einzelnen Reste der Schleimhaut haften meist sehr fest auf der Geschwulstunterlage.

Die Tumoren sind nun nicht überall gleichmässig zusawmengesetz.t. Soweit sie freilich ausserhalb des Bereiches der Niere gelegen sind, bestehen sie fast durchweg aus einem ziemlich weichen grauweissen Gewebe, in welchem fleckweise zahlreiche weite Gefässlumina sichtbar sind, und enthalten zerstreut grössere und kleinere hämorrhagische Stellen. Die Polypen, durch welche erst nach der Erhärtung in Müller'scher Flüssigkeit und Alkohol Durchsehnitte angelegt wurden, sind nicht so gleichmässig gebaut. Gehen wir bei ibrer Betrachtung von den kleineren Formen aus, so sehen wir, dass der im Calix gelegene Abschnitt von derberer Beschaffenheit ist, als der ausserhalb befindliche. Beide aber bestehen ans breiteren und schmaleren Gewebszügen, die sich. gegenseitig kreuzen und durchflechten, so dass die Schnittfäche aus der Länge und der Quere nach durchschnittenen Zügen zusammengesetzt erscheint, die in dem freien Theil der Polypen eine lockere, auf den Querschnitten fast schwammige Structur haben. Aus solchem weichen Gewebe besteht auch die oben erwähnte grosse zerfetzte Ge- 
schwulstmasse, während der grösste Polyp wesentlich derber ist. Seinen Hauptantheil bildet ein festes glänzendes weissliches Gewebe, ähnlich dem von Fibromen, und in diesem sind eingestreut weichere blutgefässreiche Knollen, von der Grösse einer Bohne bis zu der einer Wallnuss, die sich theils ringsum scharf gegen das fibröse Gewebe absetzen, theils an einzelnen Stellen oder auch in der ganzen Peripherie allmählich darin übergehen.

Die mjkroskopische Untersuchung ergiebt nun als Grundlage für den Aufhau der gesammten Geschwulstmasse ein aus kleinen Spindelzellen zusammengesetztes salcomatöses Gewebe. Dasselbe ist vielfach mit Rundzellen durchsetzt, die oft zu grösseren und kleineren Lagern zusammentreten, und reich an dünnwandigen weiten Gefässen. Stellenweise kommt es zur Bildung faseriger Intercellularsubstanz. So sind im Grossen und Ganzen die ausserhalb des Bereiches der Niere gelegenen Abschnitte gebaut. In grösserem Umfange als hier kommt es nun in den Polypen zu einer fibrösen Umwandlung des zellreichen Gewebes. In den durch ihre makroskopische festere Fügung ausgezeichneten Theilen finden wir dann ein aus geradlinigen oder gewellten derben Fasern bestehendes Bindegewebe, in welchem nur noch wenige Zellen liegen und in dieser Minsicht zeichnen sich vor Allem die filromähnlichen Partien des grössten Polypen aus.

Als besonderes Element treten nun aber in dem Gewebe der polypösen Tumoren quergestreifte Muskelfasern binzu, die in den einzelnen Geschwulstabschuitten in sehr wechselnder Menge vertheilt sind. Wan trifft sie einzeln, meist zu mehreren und zu grösseren Bündeln geordnet an. Nirgendwo aber bilden sie umfangreichere parallel verlaufende Lager, sondern stets sind sie mannichfaltig durcheinander geflochten, die einzelnen Fibrillen sowohl wie die ganzen Bündel, in den letzteren liegen die Fasern bald dicht aneinander, bald sind sie durch grössere oder geringere Mengen zelligen oder fibrösen Gewebes von einander getrennt. Die Bündel selbst liegen an manchen Stellen so dicht gedrängt, dass im Gesichtsfelde die längsgerichteten oder schrägen und quergetroffenen Durchschnitte sich berühren. Neist allerdings ist zwischen ihnen noch etwas Geschwulstgewebe vorhanden. Auch die isolirten Fibrillen durchflechten sich gewöhnlich nicht dicht gedrängt, sondern so, dass engere oder weitere $Z$ wischenräume übrig bleiben.

Am besten ausgeprägt und am reichlicbsten sind die Muskelfasern in den älteren derberen Theilen der Polypen, in den oberflächlichen weichen zerfetzten Abschnitten fehlen sie innerhalb des Spindel- oder Rundzellengewebes ganz, in den schon etwas fester gefügten zellreichen Partien, den etwas tiefer gelegenen, zumal auch den in den dorberen Theilen liegenden weicheren Heerden sind grosse Mengen von Muskelfasern nachzuweisen. Aber hier sind sie weniger weit entwickelt, viel schmaler, nicht immer deutlich quergestreift. Je jüngere Wachsthumsstufen man untersucht, desto mehr nehmen die Faserm den Charakter von sehr langen schmalen Spindelzellen an, die zunächst nur in ihren centralen Partien um den Kern eine Qnerstreifung erkennen lassen. 
Die feineren histologischen Verhältnisse dieser Muskelfasern, ihre Breite und Länge, die Ausdehnung der Querstreifung, das Verbalten des Kerns, decken sich mit den Angaben der oben genannten Autoren. Ich lasse mich daher anf diese Punkte nicht genauer ein. Aber einen UTnstand, die Beziehung nehmlich der Musculatur zu dem Spindelzellengewebe muss ich specieller besprechen. Es handelt sich da einmal um die Frage, ob als Vorstufen der Muskelfasern Spindelzellen anzusehen sind, die, erst später die Querstreifung annehmend, zunächst glatten Muskelzellen entspräehen, wie das von mehreren Seiten angegeben worden ist (s. u.). Und wenn das der Fall ist, dann fragt es sich weiterhin, ob diese Spindeln mit den Elementen des Sarcomgewebes identisch sind resp. aus ihnen hervorgehen und ob damit also alle Sarcomzellen als embryonale Muskelfasern anzusehen sind.

Ich gehe zunächst auf letzteren Punkt ein. Aus meinen Präparaten schliesse ich, dass das sarcomatöse Gewebe mit den Muskelfasern nicht in genetischem Zusammenhang steht. Es wird das schon wahrscheinlich, wenn man bedenkt, dass die fibrösen Gewebspartien aus den sareomatösen direct hervorgehen und dass die Muskelfibrillen in ersterem als abgeschlossene Züge liegen. Unter dem Mikroskop ist es freilich in den zellreichen Theilen nicht immer leicht, die Abgrenzung festzustellen. Wenn hier die Muskelbündel schon deutlich entwickelt sind, setzen sie sich allerdings genügend scharf gegen die Umgebung ab, nicht aber, wenn sie selbst nur lange und schmale Spindeln darstellen. Hier kann man nur an Uebergangspartien sicher werden, in denen man nnter ausgeprägter Entwicklung der Muskelfasern einerseits und unter Zunahme der fibrillären Grundsubstanz andererseits die Trennung der Gewebsarten sich vollziehen sieht. Es geht nlso meiner Erfahrung nach die Entwicklung der Musculatur unabbängig von der des Sarcomgewebes vor sich, dieses bildet nur den Boden, in welchem erstere wächst und demgemäss finden sich in den jüngsten und weichen Theilen der Geschwulst keine Muskelfasern, und sind sie in den derberen älteren Abschnitten am besten entwickelt.

Wenn wir somit die sarcomatöse Grundsubstanz und die eingelagerten Nuskelfasern scharf von einander trennen, so ist damit die andere Frage, ob die Entwicklung der quergestreiften Fasern stets von glatten spindeligen Elementen ausgeht, nicht berührt. Eine solche Genese lässt sich aber in grosser Ausdehnung nachweisen. Tch habe schon betont, dass die Muskelbündel in den zeilreichen, also jüngeren Abschnitten aus langen Spindelzellen sich zusammensetzen, die in der Mitte Querstreifung zu zeigen beginnen. Weiterhin findet man dann auch vollkommen glatte Elemente, die man durch Zerzupfen des Gewebes isoliren kann. Sie haben einen länglichen, oft dentlich stäbchenförmigen Kern und gleichen dann durchaus glatten Muskelfasern. Die Spindelzellen des Sarcomgewebes sind kirzer und haben einen mehr rundlichen kurzovalen Kern. Naturgemäss sind diese Unterschiede nicht so durchgreifend, dass man nun jeder einzelnen Zelle mit Sicherheit ansehen könnte, ob sie zum Mnskel- oder zum Sarcomgewebe gehorit. 


\section{7}

Zur Vervollständigung der histologischen Beschreibung müssen nun noch drei weitere Punkte besprochen werden. Der erste betrifft die Begrenzung der Polypen gegen die Nierensubstanz und letatere selbst. Es wurde schon hervorgehoben, dass die Basis der Polypen von der Niere meist durch ein lockeres Gewebe getrennt ist. Stellenweise ist allerdings die Verbindung auch eine festere. In jedem Falle aber ist histologisch die Grenze des Nierengewebes leicht festzustellen. Letzteres hat nirgendwo mehr die normale Structur, überall ist, allerdings in etwas wechselndem Umfange, eine beträchtliche Vermehrung des interstitiellen Bindegewebes zu Stande gekommen und dadurch sind die IJarnkanälcben der Marksubstanz und der Rinde weit auseinandergedrängt und, wie aus ihrer relativ geringen Anzahl zu erschliessen, offenbar zum grossen Theil untergegangen. Die noch vorbandenen geben aber hinreichende Anhaltspunkte zur Bestimmung der Grenze der Niere gegen das Geschwulstgewebe. So zeigt sich denn, entsprechend den aus dem makroskopischen Verhalten gezogenen Schlüssen, dass das Nierengewebe bei der Bildung des Tumor's vollkommen unbetheiligt ist, dass zwischen beiden nur eine analoge Verbindung existirt wie zwischen dem nornalen Nierenbecken und der Niere selbst und das's nur stellenweise, offenbar sccundär, der bindegewebige Zusammenhang ein etwas festerer geworden ist.

Der zweite Punkt bezieht sich auf das Vorkommen epithelialer Elemente in den Polypen. Solche Bildungen sind auch von mehreren der bisherigen Autoren erwähnt. Boström, sowie Kocher und Langhans beschreiben zum Theil stark dilatirte hamkanälchenartige Bildungen, theils mit, theils ohne Membrana propria. Brosin betrachtet analoge Gebilde als Lymphgefässe mit gewucherten Endothelien. Die von mir gesehenen Bilder stimmen am besten äberein mit dem, was Marchand mitgetheilt hat. Er spricht von kleinen verzweigten Schläuchen mit Cylinderepithel, stellenweise in cystische Hohlräume übergehend, unter denen. wieder einzelne mit polyedrischen Epithelzellen ausgefüllt sind. Ich sah ähnliche längliche und oft nach Árt acinöser Drüsen vielfach ausgebuchtete Räume, gleichfalls zum Theil mit desquamirten Epithel vollgepfropft. Sie besassen keine Membrana propria und waren oft dadurch, dass, wie bei Marchand, eine lebhafte Ansammlung von Rundzellen in dem umgebenden Gewebe stattgefunden hatte, peripherisch nicht mehr deutlich abzugrenzen. Solche epitheliale Bildungen habe ich nun, worauf ich noch" zurückkomme, nur in den in den Calices befindlichen Abschnitten der Polypen gefunden und zwar hier nur in den peripherischen Theilen, aber obne allen Zusammenhang mit den Harnkanälchen der benachbarten Nierensubstanz. Eine derartige genetische Beziehung ist schon nach dem gescbilderten Verbalten von Tumor und Niere unwahrscheinlich. Dazu komnt nocb, dass die epithelialen Räume in den der Niere direct anliegenden Theilen fast ganz fehlen und erst in einiger Entfernung davon zahlreicher auftreten.

Drittens bliebe noch $z u$ erwäbnen, dass sich, und zwar gleichfalls in den basalen Polypenabschnitten, kleine Gruppen von Fettzellen finden, wie sie auch in einigen der bisher beschriebenen Geschwülste vorhanden waren. 
Auf Grund der vorstehenden Mittheilungen können wir uns nunmehr wohl ein Bild der Entwickelung der gesammten Geschwulst machen. Die Neubildung ist ausgegangen von der Wand des Nierenbeckens und zwar entwickelte sich zunächst ein sarcomatöses Gewebe mit der Neigang späterhin vielfach eine fibröse Umwandlung einzugehen. Das Wachsthum geschah hauptsächlich in der Richtung gegen das Lumen des Beckens und anfänglich so, dass die Geschwulstmassen die einzelnen Kelche ausfüllten, die hier befindlichen Markkegel verdrängten, comprimirten und abflachten, um dann weiterhin durch den Hals der Calices in das gemeinsame Nierenbecken als polypöse Tumoren vorzuwuchern. Die als Grundlage der Geschwulstentwickelung anzusehende Wand des Nierenbeckens ging in dieselbe, soweit sie direct an das Nierengewebe anstösst, ganz auf und ist hier keine Spur mehr von ihr aufzufinden. Soweit andererseits das Nierenbecken von dem Fettgewebe des Hilus begrenzt wird, fand die Wucherung des Sarcoms nur in diesem und in den äusseren Schichten der Wandung selbst statt und dementsprechend ist hier, wie oben angegeben wurde, die Schleimbaut noch in einzelnen Fetzen vorhanden.

Das Verhalten des Epithels des Nierenbeckens konnte bei dem Wachsthum der polypösen Geschwülste ein doppeltes sein. Es konute die sich unter ihm entwickelnden Tumoren auch weiterhin zunächst noch überziehen und dann eventuell später durch Abstossung und mangelnden Wiederersatz verloren gehen. In der That lässt sich auf der Oberfäche der Polypen kein Epithelïberzug mehr nachweisen. Es war aber auch möglich, dass die Epithellage von dem Sarcom vielfach durchbrochen wurde und dass dieses dabei grössere und kleinere Gruppen der Zellen einschloss, die nun, da sie ja in natürlichem Zusammenhang mit der ernährenden Unterlage blieben, nicht ohne Weiteres dem Untergang geweiht waren, sondern sehr wohl an den allgemeinen Wachthumsvorgängen sich betheiligen konnten. Auf diese Weise glaube ich am einfachsten jene oben erwähnten epithelialẹn Räume zu erklären, die aus den angegebenen Gründen nicht vón Harnkanälchen abgeleitet werden konnten.

Das Gewebe der entstehenden Geschwülste bildete nun in der genauer ausgefïhrten Weise den Boden für die Entwicke- 
lung von quergestreiften Muskelfasern, die unser hauptsächliches Interesse in Anspruch nehmen. Ihr Vorkommen sucht man sich auf zwei Wegen verständlich zu machen. Einmal nimmt man mit Cohnheim an, dass bei der nahen Beziehung der Nierenanlage zu der Musculatur der hinteren Bauchwand durch eine abnorme Abschnürung leicht Theile der letzteren in jene hineingerathen und hier sich weiter entwickelnd zur Geschwulstbildung Veranlassung geben könnten. Diese Anschauung wird von den meisten Seiten gebilligt und könnte natürlich auch auf unser Object übertragen werden. Ihr gegenüber steht die andere Annahme, nach welcher die quergestreiften Fasern sich metaplastisch aus glatten Muskelfasern entwickeln.

Auf die Möglichkeit eines derartigen Vorganges, der ja in der Metaplasie zahlreicher anderer Gewebe seine Analogie ${ }^{1}$ ) findet, hat auch Marchand für seine Geschwulst hingewiesen. Er bezicht sich auf verschiedene diesbezügliche, in der Literatur niedergelegte Angaben und erinnert daran, dass Eberth in der Nierenkapsel ein Netzwerk von glatten Muskelfasern nachgewiesen und diese zur Erklärung von Myosarcomen der Niere herangezogen hat. Marchand hält es jedoch für unwahrscheinlich, dass aus diesen spärlichen Elementen die massenhafte Neubildung von Muskelsubstanz abzuleiten sei, zumal auch gegen diesen Ursprung das Wachsthum der Geschwülste spräche, welches die Niere gewissermaassen von innen her auseinanderdränge.

Nun finden sich aber ausser in der Nierenkapsel glatte Muskelfasern, und zwar in grosser Menge, auch in der Wandung des Nierenbeckens, wie vor Allem von Henle gezeigt wurde. Neuerdings hat sie Jardet ${ }^{2}$ ) nochmals ausführlich beschrieben. Somit ist, da sich unser Tumor zweifellos aus der Nierenbeckenwand entwickelt hat, die Grundlage für den metaplastischen Vorgang ausreichend gegeben. Zunächst spricht für diese Auffassung der Umstand, dass die quergestreiften Muskelfasern sich nur im Bereich des von der Niere bedeckten Abschnittes finden. Wir sahen aber, dass hier die ganze Wand des Nierenbeckens, also auch die in ihr befindliche Musculatur in die Geschwulstbildung aufgegangen ist. Im übrigen Umfange

1) Siehe Virchow über Metaplasie. Dieses Archiv Bd.97. S. 410.

2) Arch. de phys, norm. et patholog. 86. No. 2.

Archiv f. pathol. Anat. Bd. CVI. Hft 2. 
des Beckens hat der Tumor sich nur in der Umgebung desselben und in den äusseren Schichten entwickelt ohne die mit glatter Musculatur versehenen Lagen zu betheiligen. Wenn wir nun finden, dass hier quergestreifte Fasern fehlen, so spricht das mit einiger Wahrscheinlichkeit dafür, dass dort die glatten Muskelelemente die Grundlage für die Ausbildung der quergestreiften gegeben haben.

Sodann weicht die Anordnung der quergestreiften Musculatur von den gewöhnlichen Verbältnissen ab. Wir haben es nicht mit grösseren parallelfaserigen Lagern zu thun, sondern wir treffen durchgängig kleinere Bündel an, die sich ganz nach Art der glatten Musculatur in Myomen durchflechten. Die gleiche Erscheinung ist auch in den bisher beschriebenen Geschwülsten hervorgetreten und Cohnheim \%. B. hebt die Uebereinstimmung mit der Durchkreuzung der Muskelfascikel in Uterusmyomen ausdrücklich hervor.

Weiterhin kounte ich oben berichten, dass als Entwickelungsstadien der quergestreiften Elemente in den jüngeren Bündeln sich sehr lange und schmale spindelzellen finden, die glatten Muskelfasern sehr ähnlich sind. Zahlreiche entsprechende Angaben finden sich in den früheren Arbeiten. So beschreibt Marchand lange, schmale, ziemlich glänzende, an den Enden spitz zulaufende Fasern, in denen dann bei weiterer Ausbildung zunächst um den Kern eine Querstreifung des Protoplasmas auftritt. Langhans giebt an, dass er in den Bündeln neben quergestreiften Fasern auch lange Spindelzellen mit feiner Längsstreifung gesehen habe, die den glatten Muskelfasern völlig ähnlich waren. Am genauesten geht Boström auf diesen Punkt ein. Er beschreibt "schmale und breitere, oft sehr lange, an den Enden spitz zulaufende, theils etwas glänzende, theils ganz leicht längsgestreifte, spindelförmige Zellen mit einem exquisit stäbchenförmigen Kern " and fasst sie als glatte Muskelfasern auf. In ihnen sah er dann zunächst um den Kern die Querstreifung auftreten. Dieser Entwickelungsgang lässt sich mit dem Standpunkte Cohnheim's nicht gut vereinigen. Freilich stellen ja auch die quergestreiften Muskelfasern in ibren ersten embryonalen Anlagen Spindelzellen dar, aber diese unterscheiden sich durch ihren rundlich-ovalen Kern und das sehr frühzeitige Auf- 
treten der Querstreifung sehr wohl von den hier in Betracht kommenden Spindeln.

Als ferneren Grund für die Annahme eines metaplastischen Vorganges glaube ich endlich das Vorkommen von Faserbündeln anführen zu können, die aller Querstreifung entbehren, sich aber durch Uebergang in quergestreifte $\mathbf{A b}$ schnitte als Muskelelemente charakterisiren. Dass es sich hier nicht etwa um eine Degenerationserscheinung handelt, lässt sich wohl daraus schliessen, dass man in günstigen Fällen die Entwickelung der Bündel aus solchen beobachten kann, die noch in jüngeren Stadien befindlich vorwiegend aus glatten Spindeln bestehen. Die fraglichen Fasern entsprechen, abgesehen von der fehlenden Querstreifung durchaus den willkürlichen Muskelfibrillen und tragen so Eigenthümlichkeiten beider Muskelarten an sich. Auch Langhans sah solche zu Bündeln vereinigte Fibrillen, zwischen denen, wie ich auch gelegentlich wahrnehmen konnte, einzelne quergestreifte Fasern sich nachweisen liessen.

Aus allen. diesen Gründen wird die Vorstellung, dass in unserem Tumor die quergestreiften Muskelfasern sich aus glatten Elementen entwickelt haben, wie ich glaube, wahrscheinlicher als die Annahme einer embryonalen Abschnürung. Diese findet ihre Hauptstütze darin, dass die fraglichen Tumoren, wie auch in unserem Falle, als congenitale bezeichnet werden müssen. Aber dieser Umstand spricht anch keineswegs gegen eine Metaplasie. Vielmehr lässt sich sehr wohl denken, dass die noch weniger differenzirten embryonalen glatten Muskelfasern leichter zu quergestreiften werden können.

Ergänzt und gestützt wird diese Auffassung durch die Untersuchung von weiteren Geschwulstbildungen, die sich bei der Section des einige Tage nach der Operation gestorbenen Kindes ergaben. Ich lasse zunächst das abgekürzte Sectionsprotocoll folgen:

In der rechten Hälfte der Bauchwand eine längsverlaufende, wenig unterhalb des Rippenbogens beginnende, bis fast zum Beckenrande reichende, durch Nähte geschlossene laparotomische Wunde. Ihr entsprechend sind innen mit der Peritonäum einige Darmschlingen verklebt dureh einen fibrinösen Belag, der auch das übrige viscerale und parietale Peritonäum überzieht und einen Theil der Darmschlingen unter einander gleichfalls verklebt. Etwas fester adhärent sind einige Schlingen auf der Hinterwand der rechten 
Bauchhälfte, sie sind ausserdem durch die Verklebungen vielfach spitzwinklig abgeknickt und die oberhalb liegenden Darmabschnitte bis zum Magen durch Gase stark ausgedehnt.

Herz und Lungen, sowie die Milz ohne bemerkenswerthe Abnormitäten. Linke Niere anämisch, im Uebrigen wie die Nebenniere und der Ureter unverändert. Rechte Niere fehlt, an ihrer Stelle eine grosse Wundfäche, auf der jene geknickten Darmschlingen festsassen und die nach Ablösung derselben zahlreiche Unterbindungsfäden aufweist. Neben der Wirbelsäule liegen mebrere in die Wundfäche vorspringende buglige bis kleinapfelgrosse Tumoren, die auf der Schnittfläche aus einem theils gelblich-grauen, theils transparenten, gequollenen Gewebe bestehen.

Der rechte Ureter ist auf die Länge von $10 \mathrm{~cm}$ von der Blase ans gerechnet, vorhanden, aber gewaltig dilatirt, gewunden und prall. Sein oberes Ende ist durch eine Ligatur fest unterbunden. Die Ausdebnung ist bedingt durch zablreiche auf seiner Schleimbaut haftende Polypen von verschiedener Grösse. Sie sitzen gruppenweise auf etwas verdickten Wandabschnitten, fliessen gleich nach ihrem Ursprung vielfach zusammen, um sich darauf wieder in die einzelnen Theile aufzulösen. Sie sind einige Millimeter bis mebrere Centimeter lang und erreichen zum Theil die Dicke eines Katheters. Die grösseren sind in ihrem mittleren Abschnitt arn dicksten, die kleineren haben einen dünnen Stiel, der mit keulenförmiger Anschwellung endet. Eine Gruppe entspringt gleich unterhalb der' Ligaturstelle, ein Theil dieser Polypen bat kein freies Ende, sondern beftet sich $2 \mathrm{~cm}$ weiter unterhalb auf der Schleimhaut wieder fest an einer Stelle, aus der eine zweite grössere Anzahl von Geschwülsten entspringt. Drei weitere sehr lange und zarte Polypen haben ihren gemeinsamen Ursprung $2 \mathrm{~cm}$ oberhalb der Einmündung des Ureters in die Blase. Die Gesammtzahl der Polypen beträgt 22 mit Ausnahme der kleinsten. Sie besteben aus einem weichen, grösstentheils gallertig durchscheinenden, in den dickeren Abschnitten weisslichgrauen und vielfach auch mit gelben und bämorrhagischen Flecken durchsetzten Gewebe. Die von Polypen freien Absehnitte der Ureterenschleimhaut sind glatt.

Die Blasenschleimhaut glatt und blass, die Einmündungsstelle der Ureteren obne Abnormitäten.

Die histologische Untersuchung ergiebt bei den kleineren Tumoren eine den gewöhnlichen Schleimhautpolypen ähnliche Zusammensetzung, ein lockeres Bindegewebe mit grösserem oder geringerem Reichthum an Rundzellen. In den grösseren Polypen werden reichliche Spindelzellen angetroffen, die dem Gewebe vielfach einen sarcomatösen Charakter verleihen. Weiterhin finden sich dann, aber fast ausschliesslich in den basalen Abschnitten, quergestreifte Muskelfasern, die auch hier beinahe ausnahmslos bündelweise angeordnet sind. Sie erreichen nicht 
die Breite der Fibrillen in den Polypen des Nierenbeckens und auch die Bündel sind weniger umfangreich.

Zum Studium des Verhaltens der Geschwülste zur Ureterenwandung wurden Schnitte senkrecht zu letzterer angefertigt, welche diese und die basalen Theile der Tumoren umfassten. Es ergab sich, dass die circulären und längsverlaufenden Schichten glatter Musculatur des Ureters an manchen Stellen unverändert waren, an anderen aber waren die einzelnen Züge durch reichlich neugebildetes, gewöhnlich derbes Bindegewebe auseinandergedrängt, in unregelmässiger Weise, besonders gegen das angrenzende Geschwulstgewebe, verlagert. In wieder anderen Stellen fehlt die glatte Musculatur auf kürzere Strecken ganz und an ihrer Stelle befindet sich ein ziemlich zellarmes Geschwulstgewebe. Hier und an den Orten, wo eine Verlagerung der glatten Muskelbündel stattgefunden hat, ist auch aussen auf der Wand des Ureters eine flache Verdickung vorhanden, die vorwiegend aus einem faserigen, nur hier und da Rundzellen enthaltenden Bindegewebe besteht. Ausserdem sind hier gleichfalls quergestreifte Muskelfasern und jene grossen runden protoplasmatischen Zellen eingelagert, die von Marchand') und und Neumann ${ }^{2}$ ) beschrieben wurden und die als unentwickelte Fibrillen aufzufassen sind, wie auch in unserem Falle vorhandene Uebergangsformen darthun. Hier sah ich keine spindeligen den glatten Muskelfasern analoge Zellen, sehr ausgedehnt aber traf ich sie in den basalen Theilen den Polypen an. Es waren lange Spindelzellen mit ausgesprochen stäbchenförmigem Kern, häufig in Bündel zusammentretend, die ganz den in der Wand des Ureters gelegenen Zügen glatten Musculatur glichen und sich von diesen meist nur dadurch unterschieden, dass die einzelnen Spindeln etwas protoplasmatischer waren: Es liegt in der Natur der Sache, dass ich directe Uebergänge dieser aus glatten Muskelzellen bestehenden Bündel in quergestreifte nicht auffand, dazu sind sie vor Allem zu vielfach gewunden, so dass sie sich auf längere Strecken nicht verfolgen liessen. Aber neben den

1) Ueber eine Geschwulst aus quergestreiften Musfelfasern mit ungewöhnlichem Gehalte an Glycogen etc. Dieses Archiv Bd. 100 S. 42.

$\left.{ }^{2}\right)$ Ein Fall ron Myoma striocellulare am Hoden. Dieses Archiv Bd. 103 S. 497 . 
fraglichen Zügen liegen in denselben Schnitten entweder einzeln oder in bündelähnlicher Anordnung spindelige Zellen, in den die Stäbchenform des Kerns weniger deutlich ist und in deren um letzteren gelegenem Protoplasma allmählich eine Querstreifung deutlich sind. Diese Bilder sprechen für den discutirten metaplastischen Vorgang. Freilich ist ja auch hier nur das Nebeneinander maassgebend, aber wenn man die oben angeführten anderweitigen Gründen mit in Betracht zieht, gewinnt der Schluss an Berechtigung.

Die erwähnten metastatischen Tumoren neben der Wirbelsäule bestanden aus sarcomatösem Gewebe ohne alle Nuskelfasern. Fassen wir nunmehr die Ergebnisse unserer Untersuchung zusammen:

In dem colossal erweiterten Becken der bei einem vierjährigen Mädchen exstirpirten rechten Niere fanden sich zahlreiche polypöse Geschwälste, welche die Niere von innen her auseinandergedrängt und hochgradig abgeflacht hatten. Es ergab sich aus den anatomischen Verhältnissen und aus der Gegenwart ganz ähnlieher gestielter Tumoren des zugehörigen Ureters mit Sicherheit, dass jene Polypen aus der Wand des Nierenbeckens hervorgegangen waren. Sie bestanden aus einem vorwiegend sarcomatösen Grundgewebe mit zahlreichen eingelagerten quergestreiften Muskelfasern. Die Möglichkeit, dass letztere entsprechend der Vorstellung Cohnheim's auf Grund embryonaler Abschnürung sich entwickelt hatten, musste zugegeben werden. A ber verschiedene oben genauer erörterte Umstände legten die Auffassung näher, dass die quergestreiften Fasern sich metaplastisch aus den in der Wand des Nierenbeckens und des Ureters reichlich vorhandenen glatten Muskelfasern gebildet hatten, während das sarcomatöse Gewebe der Bindesubstanz der ersten Harnwege seine Entstehung verdankt.

Bei den bisher beschriebenen Sarcomen der Niere mit quergestreiften Muskelfasern hat man an einen Ursprung aus der Wand des Nierenbekens nicht gedacht, die anatomischen Befunde boten dazu keine Veranlassung. Der einzige Tumor, der 
auf Grund unseres Falles, vielleicht in ähnlicher Weise gedeutet werden könnte, ist der von Marchand beschriebene. Derselbe hatte sich vom Hilus her entwickelt und einen Theil der Niere von hier aus abgeflacht und über sich herübergespannt, ähnlich wie unsere Geschwulst die ganze Niere. Ferner ging aus der Wand des noch erhaltenen erweiterten Theiles des Beckens ein umfangreicher röthlicher weicher polypöser Körper hervor. Aber diese Anhaltspunkte sind freilich nicht ausreichend. Es kann daher erst die Untersuchung weiterer derartiger Myosarkome lehren, ob das Nierenbecken häufiger als Ursprungsstätte derselben in Betracht kommt und ob im Zusammenhang damit eine Metaplasie der vorhandenen glatten Musculatur in quergestreifte sich wahrscheinlich machen lässt. Man wird aber im Auge behalten müssen, dass die Geschwulstentwicklung auch sehr wohl gegen und in die Niere hinein erfolgen kann und dass daher auch solche Tumoren, die sich nicht durch die polypöse Form des Wachsthums und die einfache mechanische Verdrängung der Niere auszeichnen, sehr wohl aus der Nierenbeckenwand hervorgegangen sein können. 